\title{
Dysphagia as a Presenting Symptom of Myasthenia Gravis-Case Report
}

\author{
Sara Ramalho ${ }^{1}$, Sandra Pereira ${ }^{1}$, Pedro Oliveira ${ }^{1}$, Hugo Morais ${ }^{2}$, Nuno Lima ${ }^{1}$, Artur Condé ${ }^{1}$ \\ ${ }^{1}$ Otolaryngology and Head and Neck Department, Centro Hospitalar Vila Nova de Gaia/Espinho, Vila Nova de Gaia, Portugal \\ ${ }^{2}$ Neurology Department, Centro Hospitalar Vila Nova de Gaia/Espinho, Vila Nova de Gaia, Portugal \\ Email: sararamalho@gmail.com
}

Received September 23, 2013; revised October 21, 2013; accepted November 20, 2013

Copyright (C) 2014 Sara Ramalho et al. This is an open access article distributed under the Creative Commons Attribution License, which permits unrestricted use, distribution, and reproduction in any medium, provided the original work is properly cited. In accordance of the Creative Commons Attribution License all Copyrights (C) 2014 are reserved for SCIRP and the owner of the intellectual property Sara Ramalho et al. All Copyright (C) 2014 are guarded by law and by SCIRP as a guardian.

\begin{abstract}
Dysphagia is a common symptom in otolaryngology, but neuromuscular causes are rarely diagnostic hypotheses to be considered. The Myasthenia Gravis is an autoimmune disease which is characterized by muscle weakness that can affect the muscles of the face, oral cavity, pharynx or esophagus and may present with swallowing disorders. The authors present the case of a 68-year-old man who went to the emergency department of otolaryngology complaining of dysphagia. After the examination and diagnostic exams, it was diagnosed as Myasthenia Gravis. The authors conclude that the clinical suspicion should not be forgotten in order to initiate timely treatment to the effective control of the disease and complement with functional tests according to the complaints, so that it can be followed up and start rehabilitation of the patient.
\end{abstract}

\section{KEYWORDS}

Autoimmunity; Dysphagia; Myasthenia Gravis

\section{Introduction}

Dysphagia is a common symptom in Otolaryngology and there is an extensive differential diagnosis.

The Mysthenia Gravis (MG) is a rare autoimmune disease that involves muscle weakness. There are antibodies against the nicotinic receptors in the post-sinaptic neuromuscular junction of skeletal muscles. It may be associated with other autoimmune diseases such as hyperthyroidism, rheumatoid arthritis or lupus.

The disease has an estimated prevalence in the United States of about 20 cases per 100,000 inhabitants [1].

There are two types of the disease: "ocular" (corresponding to $10 \%$ of cases) [1] or "generalized" ( $90 \%$ of cases), although both may occur under different temporal evolution in the same patient.

The sex and age of onset of the disease influence the course of disease. In the age of 40 years it is more common in females and it is more prevalent in male patients aged over 50 .

The cardinal symptom is muscle weakness that is typ- ically fluctuating and fatigable, and the most common initial presentation involves a specific muscle weakness, and not a generalized weakness. Symptoms are exacerbated with exercise (it may be caused by sustained muscle action) and heat, and decreased with relaxation and cooling. The disease involves striated or voluntary muscles, with a typical anatomical distribution. The weakness is usually ocular, bulbar, proximal muscle groups (most commonly the upper limbs) and neck, but may also in some patients involve the respiratory muscles. The disease can progress from mild to moderate-severe in weeks and months with episodes of exacerbation and remission. The Myasthenia Gravis Foundation of America proposed a clinical classification of the disease into five main classes and several subclasses, according to the location of muscle weakness and its intensity. Ptosis or extraocular muscle weakness is the initial presentation in $75 \%$ of patients [1] and occurs during the course of the disease in $90 \%$ of cases (remaining exclusively ocular only $16 \%$ of cases [2-4]). Dysphagia is a common symptom in MG and it occurs in 15\% - 40\% [4] of patients 
with the generalized form of the disease, however, dysphagia as a presenting symptom is rare, occurring in 6\% [4] of cases and more rarely as the initial and unique symptom of the disease.

On physical examination, muscle strength should be thoroughly checked in multiple muscle groups to document and assess disease severity and monitor the treatment.

The MG may involve the muscles of the oropharynx, chewing and swallowing, while the palatal muscle weakness can result in rhinolalia and nasal regurgitation. The weakness of the pharyngeal muscles may result in collapse of the upper airway so that the evaluation of respiratory status is essential for evaluation of disease. It may also be the cause of dysphagia and aspiration. The weakness of the neck muscles is relatively common, affecting usually the flexor muscles. The musculature of the esophagus, including the upper esophageal sphincter (composed of striated muscle), may be affected by the disease also resulting in dysphagia. However, studies show that the esophageal muscle may be involved in its entirety. Huang et al. [5] have found the decrease in amplitude and the increase in duration of peristaltic waves around the esophagus, including the distal esophagus (smooth muscle compound).

The diagnosis of the disease involves the detection and determination of antibody titer of anti-acetylcholine receptor (AChR antibody). The test is highly specific and about $85 \%$ - 90\% of patients with generalized MG have tested positive, however, rarely, there are false negatives (particularly in the ocular form). The changes in antibody titer may reflect the progression of the disease, particularly in treatment response (although they cannot be used for monitoring therapy, because the data are not consistent). Other antibodies may be present in the diagnosis of the disease, such as anti-striated muscle or anti-muscle specific kinase (MuSK antibody). The patient should also make the assay for a rheumatoid factor, anti-nuclear antibody (ANA) and thyroid function tests to rule out other autoimmune disorders.

In addition to the serological diagnosis, the patient should also have a cervico-thoracic computer tomography to exclude thymoma. It should also be requested cranio-encephalic tomography or cranio-encephalic and orbit magnetic resonance to exclude other neurological differential diagnoses.

The electrophysiological studies are essential in the diagnosis of disease, demonstrating the defect in neuromuscular transmission. It is usually performed the repeated stimulation test (repeated stimulation of a muscle $2-3 \mathrm{~Hz}$ ) and also the single fiber electromyography, the latter being more sensitive in diagnosing MG though the former is most often used due to the ease in its realization.

There are other diagnostic tests that may be performed, such as the anticholinesterase test (with use of edrophonium).

The treatment of the disease should be individualized according to the characteristics of the patient and the severity of the disease. It may involve the use of acetylcholinesterase inhibitors with usual need to use steroid therapy in acute phases of the disease and sometimes chronic use of other immunosuppressive agents (such as azathioprine). Therapy with intravenous immunoglobulin and plasmapheresis may be held for short periods of time in situations of greater severity. Thymectomy can also be a therapeutic option, especially if there is presence of thymoma, although it may also be considered in its absence in the generalized forms of the disease.

\section{Case Report}

A 68-year-old man, arrived at the emergency department with symptoms of dysphagia for liquids and solids for two months. He also reported complaints of dysphonia for two weeks. Both symptoms worsened during the day. Physical examination on admission revealed complete eye movements without diplopia in the tests of fatigue, symmetric facial movements, symmetric elevation of the soft palate, tongue protrusion in the midline and simetrical vocal folds with no lesions. Remaining neurological examination also revealed no significant changes.

In the diagnostic process, cranio-encephalic and chest computed tomography showed no changes. The determination of antibodies to acetylcholine receptors revealed a value of $1.02 \mathrm{nmol} / \mathrm{L}$ (normal: 0.0 to $0.25 \mathrm{nmol} / \mathrm{L}$ ). The electrophoretic study and thyroid function tests were normal. The serological determination for viral hepatitis B, C and Human Immunodeficiency Virus were negative.

In electomyography there was a positive test for repeated stimulation (Figure 1).

The respiratory function study revealed mild restrictive ventilatory changes without other significant changes.

Myasthenia Gravis was diagnosed and the patient started treatment with Pyridostigmine. He didn't improve significantly, so he began treatment with human immunoglobulin for 5 days, with partial recovery and subsequent dose adjustment of Pyridostigmine. He also started low dose corticosteroids. He showed overall clinical improvement with therapy, although with few episodes of exacerbations, so he was proposed to thymectomy, which was performed with no complications.

The pathological examination revealed no thymic lesions. He is currently clinically improved, under treatment with lepicortinolo $35 \mathrm{mg} /$ day and pyridostigmine $300 \mathrm{mg} /$ day.

\section{Discussion}

Dysphagia is a rare clinical manifestation in MG as the 


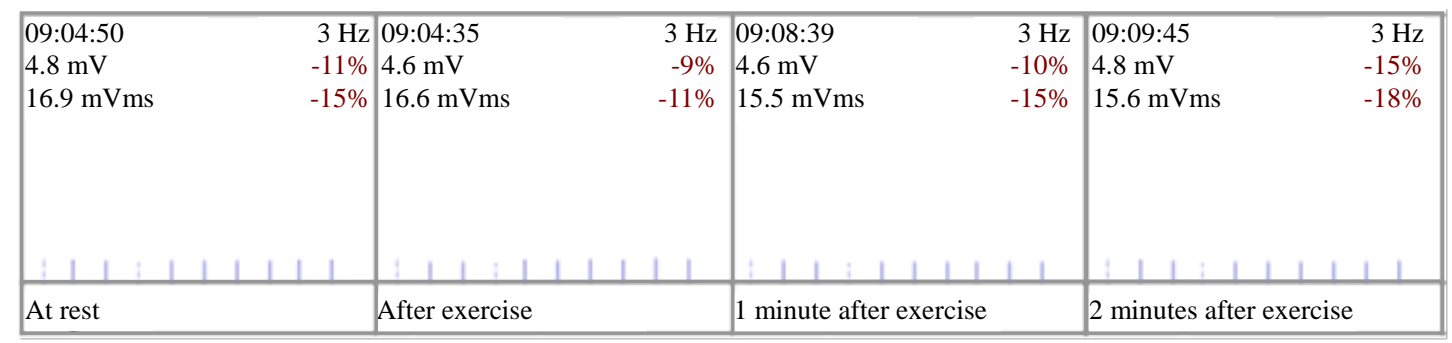

Figure 1. Repeated stimulation test-positive.

initial presentation. Considering the extensive diagnostic hypotheses involving the swallowing pathology, the neuromuscular pathology must be suspected after a medical history and physical examination, valuing the accompanying symptoms, day-time evolution of the pathology and aggravation factors.

According to electrophysiological assessments, the most affected muscles when the disease presents with dysphagia and dysarthria are the muscles of the tongue, the larynx elevators, pharyngeal constrictors, cricopharyngeal muscle [6] and the muscles of the distal esophagus [7]. The effective treatment and favorable evolution of the disease can only start after correct diagnosis and clinical assessment. In the case of clinical presentation with dysphagia, it can also be enriched with the swallowing evaluation, as videofluoroscopy [8] or swallowing endoscopy, which benefits assessment towards the correct clinical and funtional monitoring and rehabilitation.

\section{Conclusion}

MG is a diagnosis that should be suspected in the cases of progressive dysphagia. Although it is rare, this form may occur in association with other symptoms or separately. The diagnostic process should be initiated and complemented with screening tests and clinical monitoring whenever possible, in order to timely start the treatment and take control of potentially severe exacerbations of the disease.

\section{REFERENCES}

[1] A. J. Trouth, A. Dabi, N. Solieman, M. Kurukumbi and J.
Kalyanam, "Myasthenia Gravis: A Review,” Autoimmune Diseases, Vol. 2012, 2012, Article ID: 874680

[2] R. J. Romo Gonzalez, E. Chaves and H. Copello, "Dysphagia as the Sole Manifestation of Myasthenia Gravis," Acta Gastroenterologica Latinoamericana, Vol. 40, No. 2, 2010 pp. 156-158.

[3] O. Bravo Dominguez, M. Foglia Fernandez, X. Gonzalez Compte, R. Jimenez Montoya, J. Girons Bonells and M. Dicenta Sousa, "ORL Manifestations of Miasthenia Gravis,” Acta Otorrinolaringológica Española, Vol. 51, No. 6, 2000, pp. 549-551.

[4] A. Rajeshwari, G. Somayaji and S. Deviprasad, "A Rare Cause of Dysphagia: A Case Report," Indian Journal of Otolaryngology and Head \& Neck Surgery, Vol. 63, Suppl. 1, 2011, pp. 83-84. http://dx.doi.org/10.1007/s12070-011-0207-9

[5] M. H. Huang, K. L. King and K. Y. Chien, "Esophageal Manometric Studies in Patients with Myasthenia Gravis," The Journal of Thoracic and Cardiovascular Surgery, Vol. 95, 1988, pp. 281-285.

[6] C. Ertekin, N. Yuceyar and I. Aydogdu, "Clinical and Electrophysiological Evaluation of Dysphagia in Myasthenia Gravis,” Journal of Neurology, Neurosurgery \& Psychiatry, Vol. 65, No. 6, 1998, pp. 848-856. http://dx.doi.org/10.1136/jnnp.65.6.848

[7] M. Llabrés, F. J. Molina-Martinez and F. Miralles, "Dysphagia as the Sole Manifestation of Myasthenia Gravis," Journal of Neurology, Neurosurgery \& Psychiatry, Vol. 76, No. 9, 2005, pp. 1297-1300. http://dx.doi.org/10.1136/jnnp.2004.038430

[8] H.-C. Juan, I. Tou, S.-C. Lo and I.-H. Wu, "Efficacy of Postural Techniques Assessed by Videofluoroscopy for Myasthenia Gravis with Dysphagia as the Presenting Symptom: A Case Report," Journal of Medical Case Reports, Vol. 4, 2010, p. 370. http://dx.doi.org/10.1186/1752-1947-4-370 